\title{
Increased vagal tone as an isolated finding in ' patients undergoing electrophysiological testing for recurrent syncope: response to long term anticholinergic agents
}

\author{
CHRISTOPHER J MCLARAN, BERNARD J GERSH, MICHAEL J OSBORN, \\ DOUGLAS L WOOD, DECLAN D SUGRUE, DAVID R HOLMES JR, \\ STEPHEN C HAMMILL
}

From the Division of Cardiovascular Diseases and Internal Medicine, Mayo Clinic and Mayo Foundation, Rochester, Minnesota, USA

SUMMARY Features suggestive of an isolated increase in vagal tone during electrophysiological study were found in 12 patients with recurrent near syncope or syncope. Results at neurological and cardiac evaluation were otherwise normal. The increased tone or heightened sensitivity to vagal tone was manifested by abnormal atrioventricular nodal refractoriness and conduction that were reversed with atropine. The patients underwent long term treatment with an anticholinergic agent (propantheline bromide) and $75 \%$ improved. Before treatment they had experienced a median of seven episodes (range 3-28) of near syncope or syncope during 10.5 months (range 1-60). During treatment these episodes decreased to a median of one (range 0-15) during 22.5 months (range 3-67); six patients experienced no further symptoms. Three patients continued to have syncope while on treatment, and one of these required permanent cardiac pacing. No additional cause for syncope was identified in any patient.

During electrophysiological assessment of patients with syncope, evidence may be obtained pointing to an increase in vagal tone. In many of these patients treatment with anticholinergic drugs seemed to improve or eliminate the symptoms.

There have been isolated case reports of syncope being caused by excessive vagal tone in people who are otherwise healthy. In some situations if syncope occurs without the typical warning symptoms the individual may be at considerable risk. ${ }^{1-3}$

Abnormal atrioventricular nodal function is a prominent electrophysiological phenomenon that is demonstrated both during spontaneous vasovagal episodes and during electrophysiological testing in patients who have a clinical history suggestive of vasovagal syncope but who are symptom free at the time of study. ${ }^{13-5}$ Increased vagal tone, however, may also be induced by the stress of the electrophysiological study in patients with no history of near syncope or syncope.

Requests for reprints to Dr Bernard J Gersh, Mayo Clinic, 200 First Street SW, Rochester, MN 55905, USA.

Accepred for publication 24 September 1985
We have assumed that clinical episodes of near syncope or syncope in patients in whom the only identifiable abnormality is increased vagal tone during electrophysiological study were the result of the increased vagal tone. We have attempted to control the symptoms with specific anticholinergic treatment aimed at reversing the increased vagal tone. We report the long term results of this treatment in 12 patients with near syncope or syncope and isolated increased vagal tone at electrophysiological study.

\section{Patients and methods}

Patients - Twelve patients (five male and seven female) were identified who presented with recurrent near syncope or syncope; their only identifiable abnormality was increased vagal tone (defined below) at the time of electrophysiological study. Their mean age was 48 years (range 15-84 years). None of the 
patients had an identifiable cause for their symptoms despite neurological (including electroencephalography and computed tomography when indicated) and cardiac examinations (including extensive ambulatory monitoring). Associated cardiac disease included partial anomalous pulmonary venous drainage with a non-significant shunt in one patient and stable coronary artery disease in two patients. Patients were excluded from the study if they had any cardiac abnormalities that could be a cause of near syncope or syncope, including carotid sinus hypersensitivity, importanit postural hypotension (a decrease in systolic pressure of more than $20 \mathrm{~mm} \mathrm{Hg}$ on standing), and important neurological disease.

History of syncope-The patients were referred for evaluation of multiple episodes of syncope or near syncope that were causing considerable disruption to daily activities. One patient had been injured during a syncopal episode, and four other patients had experienced symptoms in situations in which their life was threatened. There was no prodrome suggestive of a vasovagal event in five of the 12 patients; all of these patients had syncope without warning. Only three patients gave a history of vasovagal events in response to painful or emotional stimuli. In eight of the patients with symptoms, syncope or near syncope was more frequent when they were standing, although three of these patients also experienced symptoms while they were sitting or lying. Four patients did not describe any specific precipitating or aggravating factors. Two patients exercised regularly, but they were not highly trained athletes.

Electrocardiographic monitoring-None of the patients had evidence suggestive of increased vagal tone on their 12 lead electrocardiogram (heart rate $<55$ beats per minute or atrioventricular conduction delay). Asymptomatic Wenckebach periodicity was noted in two patients during $\mathbf{2 4}$ hour monitoring. Definition of increased vagal tone-Increased vagal tone at the time of electrophysiological study was defined as $(a)$ atrioventricular nodal Wenckebach periodicity occurring at atrial paced rates of $\leqslant 120$ beats per minute, $(b)$ abnormally prolonged atrioventricular nodal functional and effective refractory periods ( $>502 \mathrm{~ms}$ and $>374 \mathrm{~ms}$, respectively), ${ }^{67}$ with restoration of a normal interval after the intravenous injection of atropine $(2.4 \mathrm{mg})$.

Electrophysiological study - The electrophysiological study was performed as previously described ${ }^{8}$ Antiarrhythmic agents were discontinued at least five drug half lives before evaluation. Two or three electrode catheters were introduced via the right femoral vein and positioned in the high right atrium, the atrioventricular junction, and the right ventricular apex. Atrial pacing was performed for 30 seconds at incremental rates starting just faster than sinus rhythm and increasing up to 200 beats per minute. Atrial extrastimuli were then introduced, and the $\mathbf{R}-\mathbf{R}$ interval was scanned during sinus rhythm and during atrial pacing. Heart rate and blood pressure were monitored during right carotid sinus massage and then during left carotid sinus massage. After this ventricular pacing was performed with incremental ventricular pacing for five beats until 2:1 capture or a paced cycle length of $200 \mathrm{~ms}$ was attained. This was followed by single and double extrastimuli, and the $\mathbf{R}-\mathbf{R}$ interval was scanned during sinus rhythm and ventricular pacing at 100 and 150 beats per minute from the right ventricular apex and outflow tract. The study was then repeated (without ventricular stimulation) after intravenous atropine $(2.4 \mathrm{mg})$.

Long term follow up and anticholinergic treatmentAll patients were followed up directly as outpatients or by telephone contact. During long term follow up no other cause for the symptoms was identified. The patients were treated with propantheline bromide ( 7.5 to $15 \mathrm{mg}$ three or four times daily) and the dose was adjusted to eliminate side effects. We did not believe that a placebo trial was ethical in this group of patients with profound potentially life threatening syncope.

We used Student's $t$ test for paired data for statistical analysis of the electrophysiological results. Corrected sinus node recovery time was obtained by subtracting the basic cycle length from the sinus node recovery time. The effective refractory period of the atrioventricular node was defined as the longest $A_{1} A_{2}$ measured with the His bundle electrogram that failed to conduct to the His bundle. The functional refractory period of the atrioventricular node was defined as the shortest $\mathrm{H}_{1} \mathrm{H}_{2}$ interval in

Table 1 Electrophysiological variables (mean $(S D)$ ) before and after atropine in patients with recurrent syncope

\begin{tabular}{|c|c|c|c|}
\hline Variable & Baseline & After atropine & $p$ \\
\hline $\begin{array}{l}\text { Heart rate (beats/min) } \\
\text { Corrected sinus node recovery time (ms) } \\
\text { Atrial pacing rate (beats/min) resulting in: } \\
\text { AV node Wenckebach } \\
\text { AV node FRP (ms) } \\
\text { AV node ERP (ms) }\end{array}$ & $\begin{array}{l}62(9) \\
345(167) \\
99(15) \\
616(67) \\
480(72)\end{array}$ & $\begin{array}{r}96(16) \\
189(69) \\
165(20) \\
408(49) \\
295(37)\end{array}$ & $\begin{array}{l}0.001 \\
\text { NS } \\
0.001 \\
0.001 \\
0.001\end{array}$ \\
\hline
\end{tabular}

AV, atrioventricular; ERP, effective refractory period; FRP, functional refractory period; NS, not significant. 
response to any $A_{1} A_{2}$ interval.

\section{Results}

Electrophysiological findings-Table 1 summarises the results of electrophysiological testing before and after the administration of atropine. All abnormalities of increased vagal tone (sinus rate and atrioventricular nodal conduction and refractoriness) became normal on atropine. One patient had an abnormally prolonged corrected sinus node recovery time ( $>525 \mathrm{~ms}$ ) that became normal after the administration of atropine. There were no abnormalities of His-Purkinje function or inducible ventricular arrhythmias.

Anticholinergic treatment and long term follow upAll patients received anticholinergic treatment with propantheline bromide. Before anticholinergic treatment the 12 patients had experienced a median of seven episodes (range 3-28 episodes) of near syncope or syncope during a median of 10.5 months (range 1-60 months) (Table 2). During anticholinergic treatment the near syncope or syncope decreased to a median of one episode (range 0-15 episodes) during a median of 22.5 months (range 3-67 months), and six patients experienced no further symptoms. In two patients (cases 11 and 12) the frequency of symptoms was unchanged or worse. One patient (case 10) had a reduction in the number of episodes of syncope after starting on propantheline bromide; however, he continued to experience syncope and, because of his occupation, a permanent pacemaker was placed and no further episodes of syncope were reported. In general propantheline bromide was well tolerated at a dose of $15 \mathrm{mg}$. One patient (case 9) required a reduction in the dosage to $7.5 \mathrm{mg}$ three times daily because of constipation; his symptoms remained well controlled during the next five years. Four patients complained of dry mouth, and one patient complained of blurred vision; however, these side effects were slight and the patients continued to take medication.

\section{Discussion}

The patients in this study had experienced near syncope or syncope on many occasions, and the only abnormality identified after extensive neurological and cardiac investigation was evidence of an increase in vagal tone at the time of electrophysiological testing. Coincidental pronounced variations in vagal tone will complicate the proof of a proposed correlation between the results of electrophysiological testing and clinical events. None the less, anticholinergic treatment aimed at reducing the increased vagal tone was associated with a considerable reduction in the frequency of near syncope or syncope, and this finding supports a cause and effect relation. We did not subject the overall frequency of near syncope or syncope before and after anticholinergic treatment to statistical analysis because such events are sporadic and a recent episode could have prompted medical examination. Also the occurrence of clusters of episodes of syncope could have produced a spuriously high pretreatment frequency in our patients.

Our definition of increased vagal tone at the time of electrophysiological testing is arbitrary but it is consistent with that in published reports. ${ }^{67} \mathrm{Al}$ though primary atrioventricular nodal disease could have been responsible for these electrophysiological findings and is also reversed by parasympathetic blockade, ${ }^{9}$ it is unlikely that our patients had this abnormality since they did not show progressive atrioventricular conduction abnormalities. The findings during long term follow up, during which no other causes for the near syncope or syncope emerged, and the apparent response to specific anti-

Table 2 Duration and frequency of syncopal events ${ }^{\star}$ before and during anticholinergic treatment

\begin{tabular}{|c|c|c|c|c|}
\hline \multirow[t]{2}{*}{ Case No } & \multicolumn{2}{|l|}{ Before } & \multicolumn{2}{|l|}{ During } \\
\hline & Duration (mnths) & Episodes (No) & Duration (mnths) & Episodes (No) \\
\hline $\begin{array}{l}1 \\
2 \\
3 \\
4 \\
5 \\
6 \\
7 \\
8 \\
9 \\
10 \\
11 \\
12 \\
\text { Median (range) }\end{array}$ & $\begin{array}{r}4 \\
12 \\
18 \\
36 \\
3 \\
9 \\
1 \\
48 \\
60 \\
1 \\
5 \\
12 \\
10 \cdot 5(1-60)\end{array}$ & $\begin{array}{c}13(\mathrm{~S}, \mathrm{~N}) \\
3(\mathrm{~S}) \\
8(\mathrm{~S}, \mathrm{~N}) \\
4(\mathrm{~S}) \\
6(\mathrm{~S}) \\
28(\mathrm{~S}, \mathrm{~N}) \\
3(\mathrm{~S}) \\
15(\mathrm{~S}, \mathrm{~N}) \\
25(\mathrm{~N}) \\
10(\mathrm{~S}) \\
3(\mathrm{~S}, \mathrm{~N}) \\
4(\mathrm{~S}) \\
7(3-28)\end{array}$ & $\begin{array}{r}17 \\
9 \\
67 \\
28 \\
6 \\
4 \\
36 \\
50 \\
61 \\
3 \\
46 \\
14 \\
22 \cdot 5(3-67)\end{array}$ & $\begin{array}{l}0 \\
0 \\
0 \\
0 \\
0 \\
0 \\
3(\mathrm{~N}) \\
2(\mathrm{~S}) \\
2(\mathrm{~N}) \\
4(\mathrm{~S}) \\
15(\mathrm{~N}) \\
6(\mathrm{~S}) \\
1(0-15)\end{array}$ \\
\hline
\end{tabular}

$\star N$, near syncope; $S$, syncope. 
cholinergic treatment support the suggestion that increased vagal tone was the underlying factor responsible for the presenting symptoms. Mechanisms other than increased vagal tone could have been operating, however; for example, an increased sensitivity to acetylcholine or a decreased activity of acetylcholine esterase at the nerve terminal. These factors were not investigated in this study.

Excessive resting vagal tone has been reported in some cases. These are usually young people or highly trained athletes with reversible atrioventricular block on electrocardiography. ${ }^{1011}$ They are usually symptom free, although there are reports of a few otherwise healthy individuals with recurrent and debilitating episodes of syncope and near syncope with evidence of increased vagal tone as their only clinical abnormality. ${ }^{1-4}$ The consequences of vasovagal episodes are usually not serious because there are warning symptoms before loss of consciousness. Our patients, however, experienced episodes of near syncope or syncope that occurred without provocation or were not associated with the typical prodrome of pallor, sweating, and nausea. In some situations such attacks could be life threatening, and they must limit activities such as driving and working in circumstances that are potentially hazardous.

The therapeutic options in patients with enhanced vagal tone are complicated by the fact that a vasovagal event has parasympathetic and sympathetic components-namely, bradycardia and a reduction in peripheral vascular resistance. ${ }^{1213}$ Treating the bradycardia may not necessarily improve the symptoms if there is an important additional vasodepressive component. None the less, anticholinergic treatment to prevent bradycardia was effective in 10 patients in this study and was effective in a single patient with syncope and abnormal atrioventricular refractoriness reversed by atropine described by DiMarco et al. ${ }^{5}$ Propantheline bromide was well tolerated; however, the male patients in this study were young (mean age 34 years) and unlikely to have the urinary retention that occurs in older patients on this agent.

The effectiveness of a permanent pacemaker for treating vasovagal syncope has been reported by other workers in small numbers of patients, ${ }^{2514}$ and the one patient in this study who received a pacemaker has remained symptom free. Benditt et al reported the beneficial effect of theophylline in patients with recurrent bradycardias. ${ }^{15}$ Thus, pacemakers should probably be reserved for patients with severe symptoms who do not respond adequately to theophylline or anticholinergic agents.

This study does not imply that patients who have a vasovagal episode should undergo electrophysiological testing. In most patients the history indicates the diagnosis, and loss of consciousness may be avoided by lying down when the warning symptoms occur. In patients who have recurrent vasovagal episodes that are not necessarily associated with a provocative event or that have a limited prodrome, the use of anticholinergic agents seems justified. As reported in this study patients occasionally present with recurrent near syncope or syncope with no identifiable cause. In such patients the results of electrophysiological testing may be normal except for evidence suggestive of increased vagal tone. Anticholinergic agents are worth trying in these patients.

The clinical implications of this study are important for patients who have near syncope or syncope with clinical or electrophysiological features compatible with increased vagal tone as their only identifiable abnormality. Whether this entity represents excessive vagal tone or another form of atrioventricular nodal dysfunction that becomes normal on atropine is not particularly important. In general, permanent pacemaker implantation can be avoided in these patients, and anticholinergic agents are worth trying.

This investigation was supported in part by a grant from the Institute of Cardiovascular Research, Brisbane, Australia.

\section{References}

1 Schlesinger Z, Barzilay J, Stryjer D, Almog CH. Lifethreatening "vagal reaction" to emotional stimuli. Isr $\mathcal{F}$ Med Sci 1977; 13: 59-61.

2 Strasberg B, Lam W, Swiryn S, et al. Symptomatic spontaneous paroxysmal AV nodal block due to localized hyperresponsiveness of the AV node to vagotonic reflexes. Am Heart f 1982; 103: 795-801.

3 Rasmussen V, Haunsø S, Skagen K. Cerebral attacks due to excessive vagal tone in heavily trained persons: a clinical and electrophysiologic study. Acta Med Scand 1978; 204: 401-5.

4 Sapire DW, Shah JJ, Black IFS. Prolonged atrioventricular conduction in young children and adolescents: the role of increased vagal tone. $S$ Afr Med $\mathcal{F}$ 1979; 55: 669-73.

5 DiMarco JP, Garan H, Harthorne JW, Ruskin JN. Intracardiac electrophysiologic techniques in recurrent syncope of unknown cause. Ann Intern Med 1981; 95: 542-8.

6 Denes P, Wu D, Dhingra R, Pietras RJ, Rosen KM. The effects of cycle length on cardiac refractory periods in man. Circulation 1974; 49: 32-41.

7 Schuilenburg RM, Durrer D. Conduction disturbances located within the His bundle. Circulation 1972; 45: 612-28.

8 Hammill SC, Holmes DR Jr, Wood DL, et al. Electrophysiologic testing in the upright position: improved 
evaluation of patients with rhythm disturbances using a tilt table. f Am Coll Cardiol 1984; 4: 65-71.

9 Rahilly GT, Zipes DP, Naccarelli GV, Jackman WM, Heger JJ, Prystowsky EN. Autonomic blockade in patients with normal and abnormal atrioventricular nodal function [Abstract]. Am $\mathcal{F}$ Cardiol 1982; 49: 898.

10 Lightfoot PR, Sasse L, Mandel WJ, Hayakawa H. His bundle electrograms in healthy adolescents with persistent second degree A-V block. Chest 1973; 63: 358-62.

11 Meytes. I, Kaplinsky E, Yahini JH, Hanne-Paparo N, Neufeld HN. Wenckebach A-V block: a frequent feature following heavy physical training. Am Heart $\mathcal{f}$ 1975; 90: 426-30.
12 Eckberg DL. Parasympathetic cardiovascular control in human disease: a critical review of methods and results. Am $\mathcal{F}$ Physiol 1980; 239: H581-93.

13 Glick G, Yu PN. Hemodynamic changes during spontaneous vasovagal reactions. Am $\mathcal{F}$ Med $1963 ; 34: 42-51$

14 Gulamhusein S, Naccarelli GV, Ko PT, et al. Value and limitations of clinical electrophysiologic study in assessment of patients with unexplained syncope. $A m \mathcal{F} \mathrm{Med}$ 1982; 73: 700-5.

15 Benditt DG, Benson DW Jr, Kreitt J, et al. Electrophysiologic effects of theophylline in young patients with recurrent symptomatic bradyarrhythmias. Am $\mathcal{F}$ Cardiol 1983; 52: 1223-9. 